\title{
Anti-human GITR Monoclonal Antibody AMG 228
}

National Cancer Institute

\section{Source}

National Cancer Institute. Anti-human GITR Monoclonal Antibody AMG 228. NCI

Thesaurus. Code C129694.

An agonistic anti-human glucocorticoid-induced tumor necrosis factor receptor (tumor necrosis factor receptor superfamily, member 18; TNFRSF18; GITR; CD357) humanized monoclonal antibody, with potential immune checkpoint modulating activity. Anti-human GITR monoclonal antibody AMG 228 binds to and activates GITRs found on multiple types of T-cells. This stimulates the immune system, induces both the activation and proliferation of tumor-antigen-specific T effector cells (T effs), and suppresses the function of activated $T$ regulatory cells (Tregs). This leads to immune-mediated tumor cell eradication though a cytotoxic T-lymphocyte (CTL) response. GITR, a member of the TNF receptor superfamily and T-cell receptor co-stimulator, is expressed on the surface of multiple immune cell types, including Tregs, T effs, B-cells, and natural killer (NK) cells. Inappropriately activated Tregs suppress Teffs and suppress T-cell receptor (TCR) signaling. 